\title{
Spectrum Mobility in Cognitive Radio
}

\author{
${ }^{1}$ Prof. Vinod Patil \\ ${ }^{1}$ Professor
}

Dept. of E\&Tc, Bharati Vidyapeeth

(Deemed to be University), College of Engineering, Pune, India

\author{
${ }^{2}$ Kuldeep Singh, ${ }^{2}$ Mukul Wadhe, ${ }^{2}$ Harsh Yadav \\ ${ }^{2}$ Student \\ Dept. of E\&Tc, Bharati Vidyapeeth
}

(Deemed to be University), College of Engineering, Pune, India

\begin{abstract}
Cognitive radio networks gives a powerful solution for spectrum shortage problem using powerful spectrum access. So in highly powerful network, the user other than primary communication is mostly break, spectrum mobility is a powerful tool for enabling continuous Secondary User data transfer. Namely, Secondary User performs spectrum handoff by transmission ongoing communication to a void channel. This paper provide some important points of spectrum mobility in Cognitive radio networks . Comparing of various handoff policy is considered concerning handoff latency. And important designs and related research challenges are also discussed.In this we discussed some policy of transmission their advantages and disadvantages. We also discussed current issues in spectrum mobility.And at the application and further use is discusses. We have therefore created a user interface (UI) for the successful transmission of secondary user frequency to black holes or empty holes.
\end{abstract}

Keywords-Cognitive radio; handoff policy; secondary user; spectrum mobility

\section{INTRODUCTION}

Cognitive radio networks can be defines as a network in which more than one user can shares same spectrum, at different times ,or different frequency .In this one is primary user, who register for spectrum and other are secondary user, they take permission from primary user to use the spectrum and the process by frequency or spectrum is transferred from one user to another is called spectrum mobility.

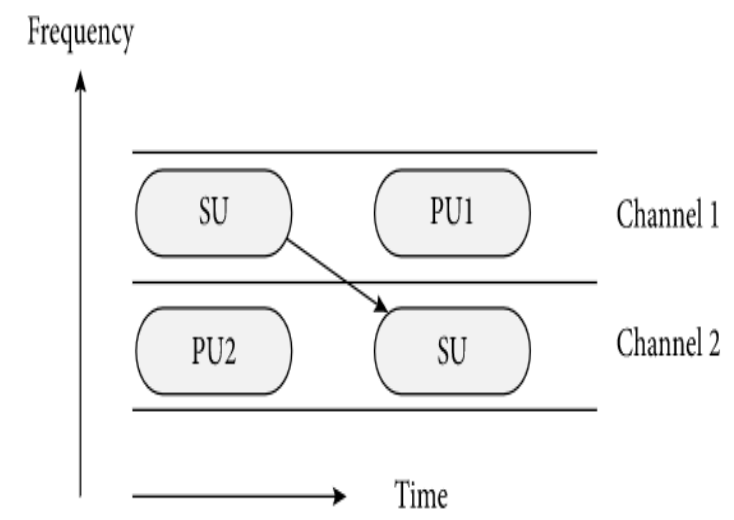

There are several other module of cognitive radio network like -:

1. Spectrum Detection- $\mathrm{n}$ this $\mathrm{CRN}$ detects for vacant frequency or holes in term of network.

2. Spectrum Decision Making - In this CRN takes decision which holes is allocated to which users.

\section{SPECTRUM HANDOFF PROCESS}

The main motive of spectrum mobility in Cognitive Radio Networks is to switching from one channel to another without breaking its connection or interruption To do this, spectrum mobility is divided into two processes: spectrum handoff and connection management. Spectrum handoff is the way of transferring ongoing data transmission from the current working channel to another void channel or hole without breaking its connection or loss of data which is being transferred. This naturally causes additional delay to Secondary User communication that directly affects Secondary User performance. To satisfy for the handoff delay, the connection management process manages and adjusts protocol parameters according to the current situation to get maximum benefit as a trade-off for higher computation effort. Thus, in this paper we discuss current issues in spectrum mobility and research challenges in spectrum Two Primary User related occasions can trigger spectrum handoff in Cognitive Radio Networks. To start with, Primary User appearance in the authorized channel fundamentally powers Secondary User to perform spectrum handoff. Second, spectrum handoff can happen due to CR user mobility. As CR users move spatially, quite possibly transmission inclusion of the Secondary User covers with a Primary User as of now utilizing a similar channel band. Being pioneering users in authorized spectrum groups, Secondary Users' movement in inheritance networks will adjust to the standard procedure: Primary Users consistently have a higher need in utilizing an authorized spectrum than Secondary Users. As a result, if Secondary User appearance makes obstruction Primary User information transmission, the Secondary User will leave the authorized channel right away.Spectrum mobility is a difficult topic for most of research activities related to diverse networks and Cognitive Radio Networks. In this paper, spectrum mobility in Cognitive Radio Networks is defined with respect to spectrum handoff. Various spectrum handoff policy are defined, judged and compared technically.

\section{CURRENT ISSUE IN SPECTRUM MOBILITY}

A. Primary User Detection - Detecting pace and exactness in spectrum detecting are two significant components for proficient spectrum mobility. Truth be told, there is a compromise between the two. Quick detecting rate would prompt less precise detecting yield. Furthermore, late spectrum detecting procedures are additionally inclined to flawed detecting results. CR hubs are 
separated into clearing gatherings and each gathering has the equivalent CDMA spreading code. On the off chance that Primary User appearance is identified, cautioning messages are spread occasionally to the whole gathering.

B. Handoff Decision - .Generally Secondary Users use spectrum overlay method where radio signs are sent with a force above Primary User clamor level. Interestingly, Secondary Users with underlay method communicate radio signs with a force beneath Primary User clamor level so both Secondary User and Primary User can utilize the equivalent authorized band simultaneously. All things considered, if the total impedance to the Primary User surpasses a specific limit, Secondary User should leave the authorized band. Consequently, spectrum handoff choice is a significant issue even in Cognitive Radio Networks with spectrum underlay strategy.

C. Target Channel Selection- Finding a reasonable objective channel over which a Secondary User can proceed with information transmission meeting is the most problem that is begging to be addressed in CR research identified with spectrum mobility. Indeed, target channel choice for spectrum handoff is a non-minor errand, since it relies upon numerous components, for example, channel limit, channel accessibility at the hour of handoff, and likelihood of divert being accessible later on. Helpless objective channel determination can cause numerous spectrum handoffs in a solitary information transmission meeting that corrupts by and large execution.

D. Routing Recovery - It is another significant issue in spectrum handoff that requires cautious arranging. Spectrum handoff is probably going to cause course breaking. In like manner, Secondary User is required to recoup the steering table to keep up network availability. Truth be told, steering recalculation is an expensive procedure as far as time and asset utilization. Consequently, steering recuperation procedure ought to be incorporated in spectrum handoff plans.

\section{SOLUTIONS IN SPECTRUM HANDOFF}

Adaptive Spectrum Handoff Strategy - Current works are generally centered around single spectrum handoff methodology. Since every spectrum handoff procedure is most appropriate for various Primary User networks, another versatile spectrum handoff calculation with numerous spectrum handoff strategy is required. In a perfect world a Secondary User should know the Primary User traffic design and apply the most appropriate hand off procedure. At the point when Primary User traffic design changes, Secondary User would see the change and adjust its hand off system likewise. In this manner, future spectrum handoff system ought to think about spectrum learning factor in the plan procedure.

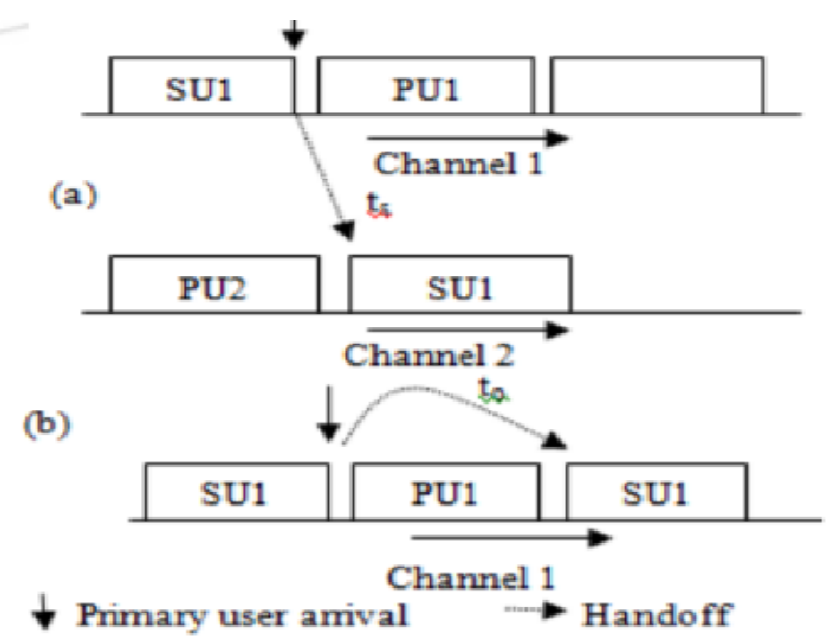

Cross Layer Link Maintenance - Connection support is at the core of spectrum mobility. The related plan issues are spread over physical, Macintosh and network layers. There front, cross layer approach between the three layers is required to address this issue productively. A large portion of the exploration works propose cross-layer arrangements, either among physical and Macintosh layers or among Macintosh and network layers. In spite of the fact that the proposed arrangements know about spectrum handoff, they are inadequate to a few degree since some significant spectrum hand off issues are ignored. For instance, steering calculations with cross-layer approach between Macintosh and network layers for the most part don't address Primary User recognition issue. Then again, Macintosh calculations with cross-layer approach between physical and Macintosh layers barely address the issue of directing recuperation. Along these lines, it is important to structure a cross-layer interface maintenance convention that would effectively address spectrum mobility issues in physical, Macintosh, and network layers to get ideal arrangement.

Energy Efficiency - In crahn, energy efficiency cy becomes a major constraint due to the limited resources of cognitive radio nodes. On the other hand, spectrum mobility methods usually rely on frequent spectrum information update and spectrum sensing evaluating the suitability of a new available spectrum band for usage and selecting the best channel among available channels could also be very tough job to do. The channel's parameters in terms of channel width, bandwidth, rate, etc. need to be evaluated before taking a decision and this could be a complex process. In a cellular architecture is assumed for CR and a spectrum aware mobility management scheme is proposed. Network architecture is introduced based on the spectrum pooling to mitigate heterogeneous spectrum availability.Another challenge is transport layer protocols'

performance of a Secondary User which could be degraded significantly as on Primary User arrival it tries to give up channel. The primary reason for most of packet losses is route failure due to mobility but TCP assumes that packet loss occurs because of congestion, so TCP will invoke congestion control mechanisms for packet loss which is actually caused by route failures, resulting in low throughput. In a solution to the above problem is given by identifying the problems which 
make TCP implementation in CR different from that of conventional wireless network. A TCP rate adapting algorithm which ensures seamless spectrum handoff as Primary User appears is proposed. New mobility and connection management approaches need to be designed to reduce delay and loss of data during spectrum handoff. A new algorithms is required to ensure that applications do not suffer from severe degradation in performance when they have to be transferred to another available frequency band due to the appearance of a primary user. This performance degradation could be due to link destruction and to preserve the quality of transmission, link maintenance is important. To maintain a required level of quality of service (QoS) for Secondary Users, an effective spectrum handoff procedure should be initiated. In a priority based model is proposed where priority is given to break user over new unbreak user. In this model the break user are required to maintain a queue and wait until all other primary and secondary users receive their services. This wait will add extra time in their delay and hence increase handoff delay and service time. By maintaining a queue we can easily identify the break user, so we can give them priority over unbreak user. This will reduce the handoff delay and maintain the required level of quality of service (QoS).

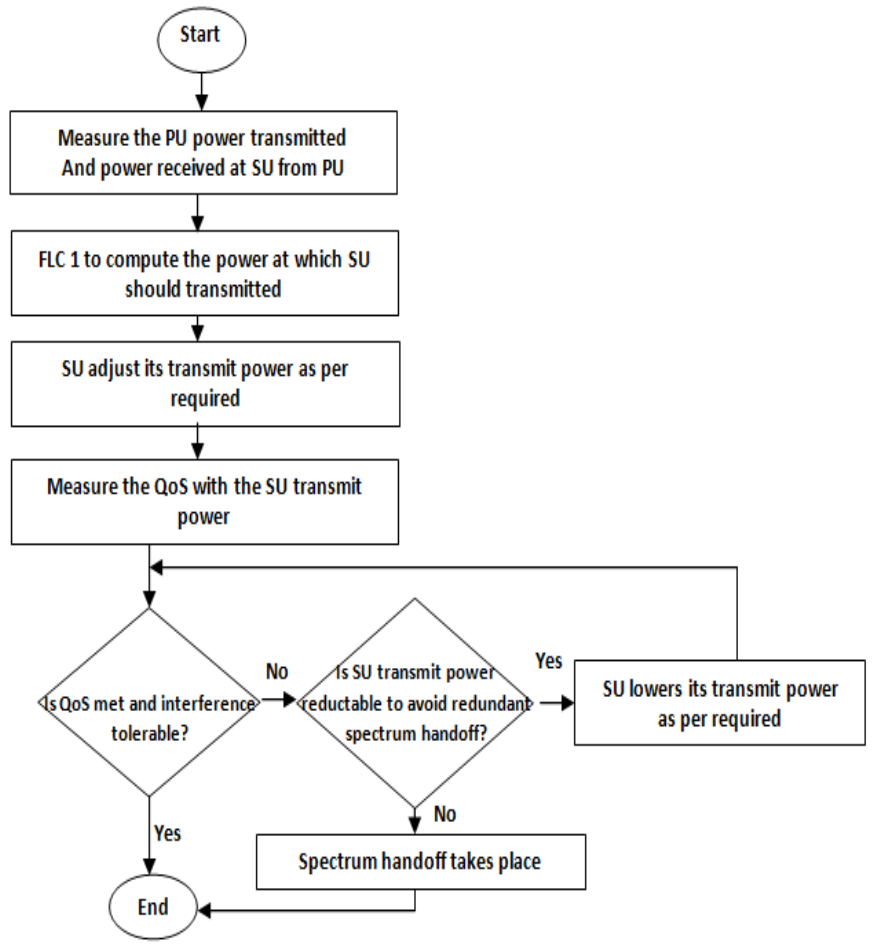

BLOCK DIAGRAM

\section{EXPIREMENTAL RESULTS}

- A graphical user interface has been made with the help Of matlab.

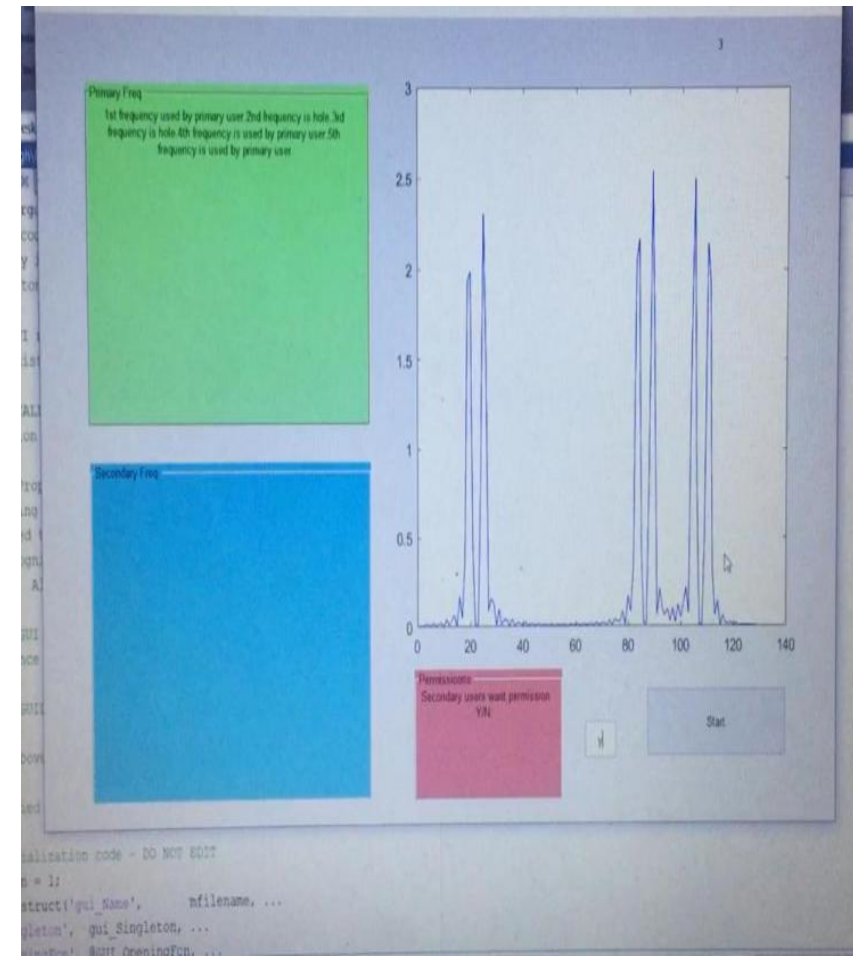

- In this $1^{\text {st }}, 4^{\text {th }}$, and $5^{\text {th }}$ waveforms are assigned to primary user and secondary user wants permission in small box right to pink.

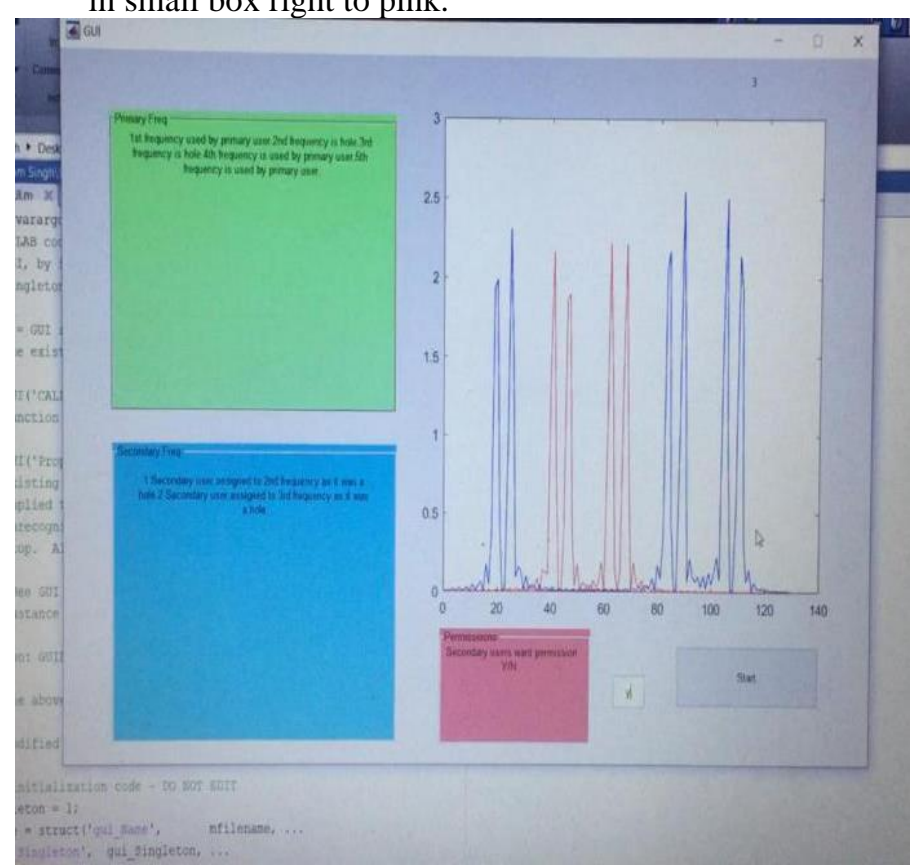

- After allowing : $2^{\text {nd }}$ frequecy assign to secondary user

And $3^{\text {rd }}$ frequency assign to another secondary user 


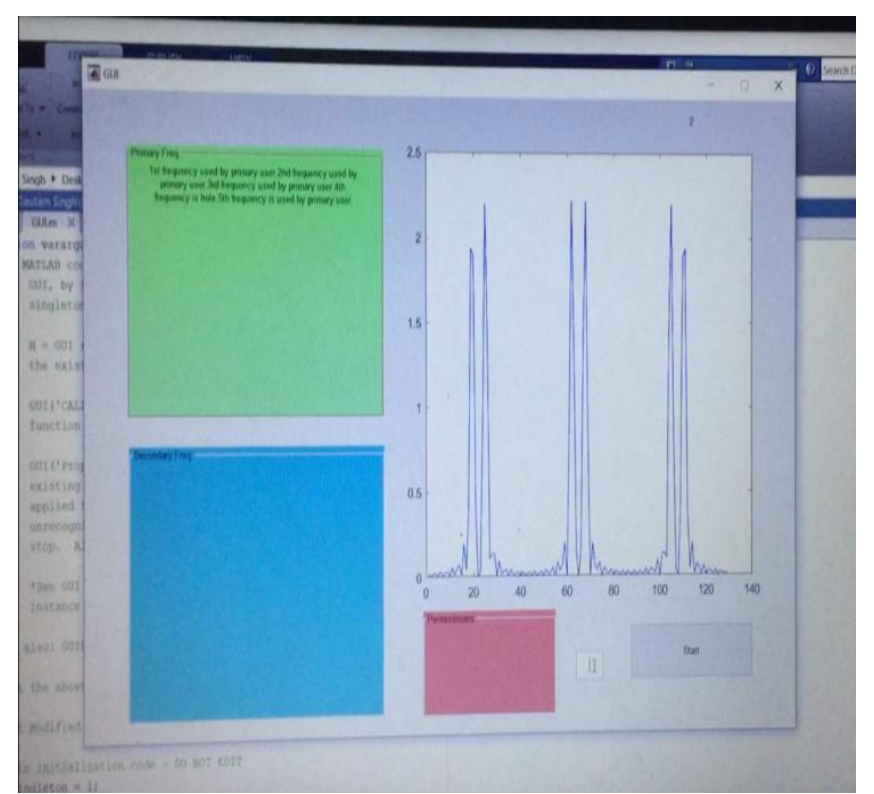

- In this $1^{\text {st }}, 3^{\text {rd }}$, and $5^{\text {th }}$ are assigned to primary user,And secondary user wants permission in small box right to pink.

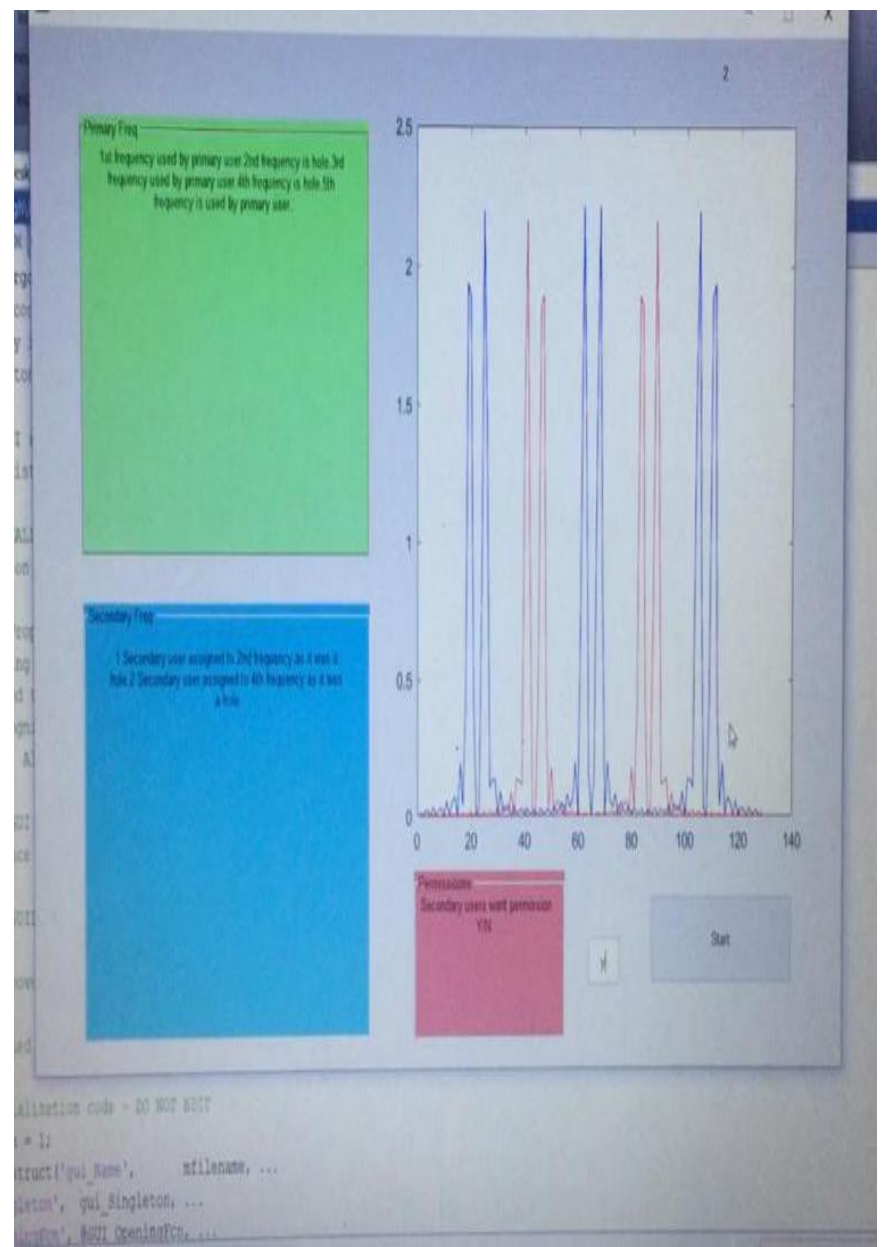

- After allowing $: 2^{\text {nd }}$ frequency assign to secondary user, $4^{\text {th }}$ frequency assign to another secondary user.

\section{APPLICATIONS}

- Efficient spectrum utilization, no wastage of frequency band

- Can be used as Public Safety in fact Justice Department of USA is working on that

- Low budget radios(two or more user can access a single spectrum).

- Automated radio resource management.

- In military frequency domains.

\section{FUTURE SCOPE}

- Direction for a replacement invention- Femtocells over TV wide spaces, the cognitive radio within the 5th Generation(5G), LTE over TV wide spaces, multimedia services over cognitive radio networks.

- Cooperative and cognitive communications

- Smart grid communications

\section{ACKNOWLEDGMENT}

This project has been possible because of the hard work and sincere efforts of not only the students but also the project guide, who helped in making the ideas clearer and also provided the necessary information relevant to the project topic. We want to show our gratitude towards Prof. Vinod Patil sir in helping us through the project, his continuous motivation and pertinent counselling helped us to achieve our goals. We would like to show our gratitude towards our institution, Bharati Vidyapeeth (DU) College of Engineering, for giving us this opportunity to work in this project and polish our skills in order to improve our knowledge. Lastly a sincere thanks to the Department of Electronics Telecommunication for giving us platforms to present our ideas and also improvising all along.

\section{REFERENCES}

[1] C.-J. Kim et al., "powerful Spectrum Access/Cognitive Radio Activities in Korea," Proc. IEEE Symp. New Frontiers in powerful Spectrum, Apr. 2010.

[2] I. F. Akyildiz, W.-Y. Lee, and K. Chowdhury, "CRAHNs: Cognitive Radio Ad Hoc Networks," Ad Hoc Networks, vol. 7, No. 5, July 2009, pp. 810.

[3] Y. Zhang, "Spectrum Handoff in Cognitive Radio Networks: Opportunistic and Negotiated Situations," IEEE Int'l. Conf. Commun. (ICC), June 2009. 